\title{
The Johnson-Lindenstrauss Lemma Almost Characterizes Hilbert Space, But Not Quite
}

\author{
William B. Johnson • Assaf Naor
}

Received: 29 July 2008 / Accepted: 12 May 2009 / Published online: 30 May 2009

(C) Springer Science+Business Media, LLC 2009

\begin{abstract}
Let $X$ be a normed space that satisfies the Johnson-Lindenstrauss lemma ( $\mathrm{J}-\mathrm{L}$ lemma, in short) in the sense that for any integer $n$ and any $x_{1}, \ldots, x_{n} \in X$, there exists a linear mapping $L: X \rightarrow F$, where $F \subseteq X$ is a linear subspace of dimension $O(\log n)$, such that $\left\|x_{i}-x_{j}\right\| \leq\left\|L\left(x_{i}\right)-L\left(x_{j}\right)\right\| \leq O(1) \cdot\left\|x_{i}-x_{j}\right\|$ for all $i, j \in\{1, \ldots, n\}$. We show that this implies that $X$ is almost Euclidean in the following sense: Every $n$-dimensional subspace of $X$ embeds into Hilbert space with distortion $2^{2^{O\left(\log ^{*} n\right)}}$. On the other hand, we show that there exists a normed space $Y$ which satisfies the J-L lemma, but for every $n$, there exists an $n$-dimensional subspace $E_{n} \subseteq Y$ whose Euclidean distortion is at least $2^{\Omega(\alpha(n))}$, where $\alpha$ is the inverse Ackermann function.
\end{abstract}

Keywords Dimension reduction · Johnson-Lindenstrauss lemma

\section{Introduction}

The J-L lemma [24] asserts that if $H$ is a Hilbert space, $\varepsilon>0, n \in \mathbb{N}$, and $x_{1}, \ldots, x_{n} \in H$, then there exists a linear mapping (even a multiple of an orthogonal projection) $L: H \rightarrow F$, where $F \subseteq H$ is a linear subspace of dimension

Research of W.B. Johnson supported in part by NSF grants DMS-0503688 and DMS-0528358. Research of A. Naor supported in part by NSF grants DMS-0528387, CCF-0635078, and CCF-0832795, BSF grant 2006009, and the Packard Foundation.

W.B. Johnson Texas A\&M University, College Station, TX 77843, USA e-mail: johnson@math.tamu.edu

A. Naor $(\bowtie)$

Courant Institute, New York, NY 10012, USA

e-mail: naor@cims.nyu.edu 
$O(c(\varepsilon) \log n)$, such that for all $i, j \in\{1, \ldots, n\}$, we have

$$
\left\|x_{i}-x_{j}\right\| \leq\left\|L\left(x_{i}\right)-L\left(x_{j}\right)\right\| \leq(1+\varepsilon)\left\|x_{i}-x_{j}\right\| .
$$

This fact has found many applications in mathematics and computer science, in addition to the original application in [24] to a Lipschitz extension problem. The widespread applicability of the J-L lemma in computer science can be (somewhat simplistically) attributed to the fact that it can be viewed as a compression scheme which helps to reduce significantly the space required for storing multidimensional data. We shall not attempt to list here all the applications of the $\mathrm{J}-\mathrm{L}$ lemma to areas ranging from nearest neighbor search to machine learning - we refer the interested reader to $[1,18-20,27,28,43]$ and the references therein for a partial list of such applications.

The applications of (1) involve various requirements from the mapping $L$. While some applications just need the distance preservation condition (1) and not the linearity of $L$, most applications require $L$ to be linear. Also, many applications are based on additional information that comes from the proof of the $\mathrm{J}-\mathrm{L}$ lemma, such as the fact that $L$ arises with high probability from certain distributions over linear mappings. The linearity of $L$ is useful, for example, for fast evaluation of the images $L\left(x_{i}\right)$, and also because these images behave well when additive noise is applied to the initial vectors $x_{1}, \ldots, x_{n}$.

Due to the usefulness of the J-L lemma, there has been considerable effort by researchers to prove such a dimensionality reduction theorem in other normed spaces. All of these efforts have thus far resulted in negative results which show that the $\mathrm{J}-\mathrm{L}$ lemma fails to hold true in certain non-Hilbertian settings. In [13] Charikar and Sahai proved that there is no dimension reduction via linear mappings in $L_{1}$. This negative result was extended to any $L_{p}, p \in[1, \infty] \backslash\{2\}$, by Lee, Mendel, and Naor [30]. Negative results for dimension reduction without the requirement that the embedding $L$ is linear are known only for the spaces $L_{1}[9,30,31]$ and $L_{\infty}[3,7,25,30,33]$. Here we show that the negative results for linear dimension reduction in $L_{p}$ spaces are a particular case of a much more general phenomenon: A normed space that satisfies the $\mathrm{J}-\mathrm{L}$ lemma is very close to being Euclidean in the sense that all of its $n$-dimensional subspaces are isomorphic to Hilbert space with distortion $2^{2^{O\left(\log ^{*}(n)\right)}}$. Here and in what follows, if $x \geq 1$, then $\log ^{*}(x)$ is the unique integer $k$ such that if we define $a_{1}=1$ and $a_{i+1}=e^{\bar{a}_{i}}$ (i.e., $a_{i}$ is an exponential tower of height $i$ ), then $a_{k}<x \leq a_{k+1}$.

In order to state our results we recall the following notation: The Euclidean distortion of a finite-dimensional normed space $X$, denoted $c_{2}(X)$, is the infimum over all $D>0$ such that there exists a linear mapping $S: X \rightarrow \ell_{2}$ which satisfies $\|x\| \leq\|S(x)\| \leq D\|x\|$ for all $x \in X$. Note that in the computer science literature the notation $c_{2}(X)$ deals with bi-Lipschitz embeddings, but in the context of normed spaces it can be shown that the optimal bi-Lipschitz embedding may be chosen to be linear (this is explained, for example, in [6, Chap. 7]). The parameter $c_{2}(X)$ is also known as the Banach-Mazur distance between $X$ and Hilbert space.

Theorem 1.1 For every $D, K>0$, there exists a constant $c=c(K, D)>0$ with the following property. Let $X$ be a Banach space such that for every $n \in \mathbb{N}$ and every 
$x_{1}, \ldots, x_{n} \in X$, there exists a linear subspace $F \subseteq X$, of dimension at most $K \log n$, and a linear mapping $S: X \rightarrow F$ such that $\left\|x_{i}-x_{j}\right\| \leq\left\|S\left(x_{i}\right)-S\left(x_{j}\right)\right\| \leq D \| x_{i}-$ $x_{j} \|$ for all $i, j \in\{1, \ldots, n\}$. Then for every $k \in \mathbb{N}$ and every $k$-dimensional subspace $E \subseteq X$, we have

$$
c_{2}(E) \leq 2^{2^{c \log ^{*}(k)}} .
$$

The proof of Theorem 1.1 builds on ideas from [13, 30], while using several fundamental results from the local theory of Banach spaces. Namely, in [30] the $L_{1}$ point-set from [13] was analyzed via an analytic argument which extends to any $L_{p}$ space, $p \neq 2$, rather than the linear programming argument in [13]. In Sect. 2 we construct a variant of this point-set in any Banach space and use it in conjunction with some classical results in Banach space theory to prove Theorem 1.1.

The fact that the bound on $c_{2}(E)$ in (2) is not $O(1)$ is not just an artifact of our iterative proof technique: There do exist non-Hilbertian Banach spaces which satisfy the J-L lemma!

Theorem 1.2 There exist two universal constants $D, K>0$ and a Banach space $X$ such that for every $n \in \mathbb{N}$ and every $x_{1}, \ldots, x_{n} \in X$, there exists a linear subspace $F \subseteq X$, of dimension at most $K \log n$, and a linear mapping $S: X \rightarrow F$ such that $\left\|x_{i}-x_{j}\right\| \leq\left\|S\left(x_{i}\right)-S\left(x_{j}\right)\right\| \leq D\left\|x_{i}-x_{j}\right\|$ for all $i, j \in\{1, \ldots, n\}$. Moreover, for every integer $n$, the space $X$ has an $n$-dimensional subspace $F_{n} \subseteq X$ with

$$
c_{2}\left(F_{n}\right) \geq 2^{c \alpha(n)},
$$

where $c>0$ is a universal constant, and $\alpha(n) \rightarrow \infty$ is the inverse Ackermann function.

We refer the readers to Sect. 3 for the definition of the inverse Ackermann function. The Banach space $X$ in Theorem 1.2 is the 2-convexification of the Tsirelson space [14], denoted $T^{(2)}$, which we shall now define. The definition below, due to Figiel and Johnson [16], actually gives the dual to the space constructed by Tsirelson (see the book [12] for a comprehensive discussion). Let $c_{00}$ denote the space of all finitely supported sequences of real numbers. The standard unit basis of $c_{00}$ is denoted by $\left\{e_{i}\right\}_{i=1}^{\infty}$. Given $A \subseteq \mathbb{N}$, we denote by $P_{A}$ the restriction operator to $A$, i.e., $P_{A}\left(\sum_{i=1}^{\infty} x_{i} e_{i}\right)=\sum_{i \in A} x_{i} e_{i}$. Given two finite subsets $A, B \subseteq \mathbb{N}$, we write $A<B$ if $\max A<\min B$. Define inductively a sequence of norms $\left\{\|\cdot\|_{m}\right\}_{m=0}^{\infty}$ by $\|x\|_{0}=\|x\|_{c_{0}}=\max _{j \geq 1}\left|x_{j}\right|$ and

$$
\begin{gathered}
\|x\|_{m+1}=\max \left\{\|x\|_{m}, \frac{1}{2} \sup \left\{\sum_{j=1}^{n}\left\|P_{A_{j}}(x)\right\|_{m}: n \in \mathbb{N}, A_{1}, \ldots, A_{n} \subseteq \mathbb{N}\right. \text { finite, }\right. \\
\left.\left.\{n\}<A_{1}<A_{2}<\cdots<A_{n}\right\}\right\} .
\end{gathered}
$$

Then for each $x \in c_{00}$, the sequence $\left\{\|x\|_{m}\right\}_{m=0}^{\infty}$ is nondecreasing and bounded from above by $\|x\|_{\ell_{1}}=\sum_{j=1}^{\infty}\left|x_{j}\right|$. It follows that the limit $\|x\|_{T}:=\lim _{m \rightarrow \infty}\|x\|_{m}$ exists. 
The space $X=T^{(2)}$ from Theorem 1.2 is the completion of $c_{00}$ under the norm

$$
\left\|\sum_{j=1}^{\infty} x_{j} e_{j}\right\|_{T^{(2)}}:=\left\|\sum_{j=1}^{\infty}\left|x_{j}\right|^{2} e_{j}\right\|_{T}^{1 / 2}
$$

The proof of the fact that $T^{(2)}$ satisfies the J-L lemma consists of a concatenation of several classical results, some of which are quite deep. The lower bound (3) follows from the work of Bellenot [5]. The details are presented in Sect. 3.

\section{Proof of Theorem 1.1}

Let $(X,\|\cdot\|)$ be a normed space. The Gaussian type 2 constant of $X$, denoted $T_{2}(X)$, is the infimum over all $T>0$ such that for every $n \in \mathbb{N}$ and every $x_{1}, \ldots, x_{n} \in X$, we have

$$
\mathbb{E}\left\|\sum_{i=1}^{n} g_{i} x_{i}\right\|^{2} \leq T^{2} \sum_{i=1}^{n}\left\|x_{i}\right\|^{2}
$$

Here, and in what follows, $g_{1}, \ldots, g_{n}$ denote i.i.d. standard Gaussian random variables. The cotype 2 constant of $X$, denoted $C_{2}(X)$, is the infimum over all $C>0$ such that for every $n \in \mathbb{N}$ and every $x_{1}, \ldots, x_{n} \in X$, we have

$$
\sum_{i=1}^{n}\left\|x_{i}\right\|^{2} \leq C^{2} \mathbb{E}\left\|\sum_{i=1}^{n} g_{i} x_{i}\right\|^{2}
$$

A famous theorem of Kwapien [29] (see also the exposition in [36, Theorem 3.3]) states that

$$
c_{2}(X) \leq T_{2}(X) \cdot C_{2}(X) .
$$

An important theorem of Tomczak-Jaegermann [40] states that if the Banach space $X$ is $d$-dimensional, then there exist $x_{1}, \ldots, x_{d}, y_{1} \ldots, y_{d} \in X \backslash\{0\}$ for which

$\mathbb{E}\left\|\sum_{i=1}^{d} g_{i} x_{i}\right\|^{2} \geq \frac{T_{2}(X)^{2}}{2 \pi} \sum_{i=1}^{d}\left\|x_{i}\right\|^{2}$ and $\sum_{i=1}^{d}\left\|y_{i}\right\|^{2} \geq \frac{C_{2}(X)^{2}}{2 \pi} \mathbb{E}\left\|\sum_{i=1}^{d} g_{i} y_{i}\right\|^{2}$.

In other words, for $d$-dimensional spaces, it suffices to consider $n=d$ in (6) and (7) in order to compute $T_{2}(X)$ and $C_{2}(X)$ up to a universal factor. For our purposes, it suffices to use the following simpler fact due to Figiel, Lindenstrauss, and Milman [17, Lemma 6.1]: If $\operatorname{dim}(X)=d$, then there exist $x_{1}, \ldots, x_{d(d+1) / 2}, y_{1}, \ldots, y_{d(d+1) / 2} \in$ $X \backslash\{0\}$ for which

$$
\mathbb{E}\left\|\sum_{i=1}^{d(d+1) / 2} g_{i} x_{i}\right\|^{2}=T_{2}(X)^{2} \sum_{i=1}^{d(d+1) / 2}\left\|x_{i}\right\|^{2} \text { and }
$$




$$
\sum_{i=1}^{d(d+1) / 2}\left\|y_{i}\right\|^{2}=C_{2}(X)^{2} \mathbb{E}\left\|\sum_{i=1}^{d(d+1) / 2} g_{i} y_{i}\right\|^{2} .
$$

We note, however, that it is possible to improve the constant terms in Theorem 1.1 if we use (9) instead of (10) in the proof below. We shall now sketch the proof of (10), taken from [17, Lemma 6.1], since this type of finiteness result is used crucially in our proof of Theorem 1.1.

We claim that if $m>d(d+1) / 2$ and $u_{1}, \ldots, u_{m} \in X$, then there are $v_{1}, \ldots, v_{m-1}$, $w_{1}, \ldots, w_{m-1} \in X$ such that

$$
\begin{aligned}
& \mathbb{E}\left\|\sum_{i=1}^{m-1} g_{i} v_{i}\right\|^{2}+\mathbb{E}\left\|\sum_{i=1}^{m-1} g_{i} w_{i}\right\|^{2}=\mathbb{E}\left\|\sum_{i=1}^{m} g_{i} u_{i}\right\|^{2} \text { and } \\
& \sum_{i=1}^{m-1}\left\|v_{i}\right\|^{2}+\sum_{i=1}^{m-1}\left\|w_{i}\right\|^{2}=\sum_{i=1}^{m}\left\|u_{i}\right\|^{2} .
\end{aligned}
$$

Note that (11) clearly implies (10) since it shows that in the definitions (6) and (7) we can take $n=d(d+1) / 2$ (in which case the infima in these definitions are attained by a simple compactness argument).

To prove (11) we can think of $X$ as $\mathbb{R}^{d}$ equipped with a norm $\|\cdot\|$. The random vector $\sum_{i=1}^{m} g_{i} u_{i}=\left(\sum_{i=1}^{d} g_{i} u_{i j}\right)_{j=1}^{d}$ has a Gaussian distribution with covariance matrix $A=\left(\sum_{i=1}^{m} u_{i j} u_{i k}\right)_{j, k=1}^{d}=\sum_{i=1}^{m} u_{i} \otimes u_{i}$. Thus the symmetric matrix $A$ is in the cone generated by the symmetric matrices $\left\{u_{i} \otimes u_{i}\right\}_{i=1}^{m}$. By Caratheodory's theorem for cones (see, e.g., [15]) we may reorder the vectors $u_{i}$ so as to find scalars $c_{1} \geq c_{2} \geq \cdots \geq c_{m} \geq 0$ with $c_{i}=0$ for $i>d(d+1) / 2$, such that $A=$ $\sum_{i=1}^{m} c_{i} u_{i} \otimes u_{i}$. This sum contains at most $d(d+1) / 2$ nonzero summands. Define $v_{i}:=\sqrt{c_{i} / c_{1}} \cdot u_{i}$ (so that there are at most $d(d+1) / 2 \leq m-1$ nonzero $v_{i}$ ) and $w_{i}=\sqrt{1-c_{i} / c_{1}} \cdot u_{i}$ (so that $w_{1}=0$ ). The second identity in (11) is trivial with these definitions. Now, the random vector $\sum_{i=1}^{m} g_{i} v_{i}$ has covariance matrix $\frac{1}{c_{1}} \sum_{i=1}^{m} c_{i} u_{i} \otimes u_{i}=\frac{1}{c_{1}} A$, and the random vector $\sum_{i=1}^{m} g_{i} w_{i}$ has covariance matrix $\sum_{i=1}^{m}\left(1-c_{i} / c_{1}\right) u_{i} \otimes u_{i}=\left(1-1 / c_{1}\right) A$. Thus $\mathbb{E}\left\|\sum_{i=1}^{m-1} g_{i} v_{i}\right\|^{2}=\frac{1}{c_{1}} \mathbb{E}\left\|\sum_{i=1}^{m} g_{i} u_{i}\right\|^{2}$ and $\mathbb{E}\left\|\sum_{i=1}^{m-1} g_{i} w_{i}\right\|^{2}=\left(1-1 / c_{1}\right) \mathbb{E}\left\|\sum_{i=1}^{m} g_{i} u_{i}\right\|^{2}$. This completes the proof of (11).

We are now in position to prove Theorem 1.1. Define

$$
\Delta(n):=\Delta_{X}(n):=\sup \left\{c_{2}(F): F \subseteq X \text { linear subspace, } \operatorname{dim}(F) \leq n\right\} .
$$

Note that by John's theorem [21] (see also the beautiful exposition in [4]) $\Delta(n) \leq \sqrt{n}$. Our goal is to obtain a much better bound on $\Delta(n)$. To this end let $F \subseteq X$ be a linear subspace of dimension $k \leq n$. Let $m$ be the integer satisfying $2^{m-1}<k(k+1) / 2 \leq$ $2^{m}$. We shall use the vectors from (10). By adding some zero vectors so as to have $2^{m}$ vectors and labeling them (for convenience) by the subsets of $\{1, \ldots, m\}$, we obtain $\left\{x_{A}\right\}_{A \subseteq\{1, \ldots, m\}},\left\{y_{A}\right\}_{A \subseteq\{1, \ldots, m\}} \subseteq X$ such that

$$
\mathbb{E}\left\|\sum_{A \subseteq\{1, \ldots, m\}} g_{A} x_{A}\right\|^{2}=T_{2}(F)^{2} \sum_{A \subseteq\{1, \ldots, m\}}\left\|x_{A}\right\|^{2}>0,
$$




$$
\sum_{A \subseteq\{1, \ldots, m\}}\left\|y_{A}\right\|^{2}=C_{2}(F)^{2} \mathbb{E}\left\|\sum_{A \subseteq\{1, \ldots, m\}} g_{A} y_{A}\right\|^{2}>0
$$

For every $\varepsilon=\left(\varepsilon_{1}, \ldots, \varepsilon_{m}\right) \in\{-1,1\}$ and $A \subseteq\{1, \ldots, m\}$, consider the Walsh function $W_{A}(\varepsilon)=\prod_{i \in A} \varepsilon_{i}$. For every $g=\left\{g_{A}\right\}_{A \subseteq\{1, \ldots, m\}}$, define $\Phi_{g}, \Psi_{g}:\{-1,1\}^{m} \rightarrow F$ by

$$
\Phi_{g}(\varepsilon):=\sum_{A \subseteq\{1, \ldots, m\}} g_{A} W_{A}(\varepsilon) x_{A} \quad \text { and } \quad \Psi_{g}(\varepsilon):=\sum_{A \subseteq\{1, \ldots, m\}} g_{A} W_{A}(\varepsilon) y_{A} .
$$

Thus $\Phi_{g}, \Psi_{g}$ are random $F$-valued functions given by the random Fourier expansions in (15) — the randomness is with respect to the i.i.d. Gaussians $g=\left\{g_{A}\right\}_{A \subseteq\{1, \ldots, m\}}$. These random functions induce the following two random subsets of $F$ :

$$
\begin{aligned}
U_{g} & :=\left\{\Phi_{g}(\varepsilon)\right\}_{\varepsilon \in\{-1,1\}^{m}} \cup\left\{x_{A}\right\}_{A \subseteq\{1, \ldots, m\}} \cup\{0\} \quad \text { and } \\
V_{g} & :=\left\{\Psi_{g}(\varepsilon)\right\}_{\varepsilon \in\{-1,1\}^{m}} \cup\left\{y_{A}\right\}_{A \subseteq\{1, \ldots, m\}} \cup\{0\} .
\end{aligned}
$$

Then $\left|U_{g}\right|,\left|V_{g}\right| \leq 2^{m+1}+1 \leq 2 k(k+1)+1 \leq 2(n+1)^{2}$. By the assumptions of Theorem 1.1 it follows that there exist two subspaces $E_{g}, E_{g}^{\prime} \subseteq X$ with $\operatorname{dim}\left(E_{g}\right), \operatorname{dim}\left(E_{g}^{\prime}\right) \leq K \log \left(2(n+1)^{2}\right) \leq 4 K \log (n+1)$ and two linear mappings $L_{g}: X \rightarrow E_{g}, L_{g}^{\prime}: X \rightarrow E_{g}^{\prime}$ which satisfy

$$
x, y \in U_{g} \quad \Longrightarrow \quad\|x-y\| \leq\left\|L_{g}(x)-L_{g}(y)\right\| \leq D\|x-y\|
$$

and

$$
x, y \in V_{g} \quad \Longrightarrow \quad\|x-y\| \leq\left\|L_{g}^{\prime}(x)-L_{g}^{\prime}(y)\right\| \leq D\|x-y\| .
$$

Moreover, by the definition of $\Delta(\cdot)$ there are two linear mappings $S_{g}: E_{g} \rightarrow \ell_{2}$ and $S_{g}^{\prime}: E_{g}^{\prime} \rightarrow \ell_{2}$ which satisfy

$$
x \in E_{g} \quad \Longrightarrow \quad\|x\| \leq\left\|S_{g}(x)\right\|_{2} \leq 2 \Delta(4 K \log (n+1))\|x\|
$$

and

$$
x \in E_{g}^{\prime} \quad \Longrightarrow \quad\|x\| \leq\left\|S_{g}^{\prime}(x)\right\|_{2} \leq 2 \Delta(4 K \log (n+1))\|x\| .
$$

By the orthogonality of the Walsh functions we see that

$$
\begin{aligned}
\mathbb{E}_{\varepsilon}\left\|S_{g}\left(L_{g}\left(\Phi_{g}(\varepsilon)\right)\right)\right\|_{2}^{2} & =\mathbb{E}_{\varepsilon}\left\|\sum_{A \subseteq\{1, \ldots, m\}} g_{A} W_{A}(\varepsilon) S_{g}\left(L_{g}\left(x_{A}\right)\right)\right\|_{2}^{2} \\
& =\sum_{A \subseteq\{1, \ldots, m\}} g_{A}^{2}\left\|S_{g}\left(L_{g}\left(x_{A}\right)\right)\right\|_{2}^{2}
\end{aligned}
$$


and

$$
\begin{aligned}
\mathbb{E}_{\varepsilon}\left\|S_{g}^{\prime}\left(L_{g}\left(\Psi_{g}(\varepsilon)\right)\right)\right\|_{2}^{2} & =\mathbb{E}_{\varepsilon}\left\|\sum_{A \subseteq\{1, \ldots, m\}} g_{A} W_{A}(\varepsilon) S_{g}\left(L_{g}\left(y_{A}\right)\right)\right\|_{2}^{2} \\
& =\sum_{A \subseteq\{1, \ldots, m\}} g_{A}^{2}\left\|S_{g}\left(L_{g}\left(y_{A}\right)\right)\right\|_{2}^{2} .
\end{aligned}
$$

A combination of the bounds in (16) and (18) shows that for all $A \subseteq\{1, \ldots, m\}$, we have $\left\|S_{g}\left(L_{g}\left(x_{A}\right)\right)\right\|_{2} \leq 2 D \Delta(4 K \log (n+1))\left\|x_{A}\right\|$ and for all $\varepsilon \in\{-1,1\}^{m}$, we have $\left\|S_{g}\left(L_{g}\left(\Phi_{g}(\varepsilon)\right)\right)\right\|_{2} \geq\left\|\Phi_{g}(\varepsilon)\right\|$. Thus (20) implies that

$$
\mathbb{E}_{\varepsilon}\left\|\Phi_{g}(\varepsilon)\right\|^{2} \leq 4 D^{2} \Delta(4 K \log (n+1))^{2} \sum_{A \subseteq\{1, \ldots, m\}} g_{A}^{2}\left\|x_{A}\right\|^{2} .
$$

Arguing similarly, while using (17), (19), and (21), we see that

$$
\mathbb{E}_{\varepsilon}\left\|\Psi_{g}(\varepsilon)\right\|^{2} \geq \frac{1}{4 D^{2} \Delta(4 K \log (n+1))^{2}} \sum_{A \subseteq\{1, \ldots, m\}} g_{A}^{2}\left\|y_{A}\right\|^{2} .
$$

Taking expectation with respect to the Gaussians $\left\{g_{A}\right\}_{A \subseteq\{1, \ldots, m\}}$ in (22), we see that

$$
\begin{aligned}
4 D^{2} \Delta(4 K \log (n+1))^{2} \sum_{A \subseteq\{1, \ldots, m\}}\left\|x_{A}\right\|^{2} & \geq \mathbb{E}_{g} \mathbb{E}_{\varepsilon}\left\|\sum_{A \subseteq\{1, \ldots, m\}} g_{A} W_{A}(\varepsilon) x_{A}\right\|^{2} \\
& =\mathbb{E}_{g}\left\|\sum_{A \subseteq\{1, \ldots, m\}} g_{A} x_{A}\right\|^{2}
\end{aligned}
$$

where we used the fact that for each fixed $\varepsilon \in\{-1,1\}^{m}$, the random variables $\left\{W_{A}(\varepsilon) g_{A}\right\}_{A \subseteq\{1, \ldots, m\}}$ have the same joint distribution as the random variables $\left\{g_{A}\right\}_{A \subseteq\{1, \ldots, m\}}$. Similarly, taking expectation in (23) yields

$$
\sum_{A \subseteq\{1, \ldots, m\}}\left\|y_{A}\right\|^{2} \leq 4 D^{2} \Delta(4 K \log (n+1))^{2} \mathbb{E}_{g}\left\|\sum_{A \subseteq\{1, \ldots, m\}} g_{A} y_{A}\right\|^{2} .
$$

Combining (24) with (13) and (25) with (14), we get the bounds

$$
T_{2}(F), C_{2}(F) \leq 2 D \Delta(4 K \log (n+1)) .
$$

In combination with Kwapien's theorem (8) we deduce that

$$
c_{2}(F) \leq T_{2}(F) C_{2}(F) \leq 4 D^{2} \Delta(4 K \log (n+1))^{2} .
$$

Since $F$ was an arbitrary subspace of $X$ of dimension at most $n$, it follows that

$$
\Delta(n) \leq 4 D^{2} \Delta(4 K \log (n+1))^{2} .
$$


Iterating (26) $\log ^{*}(n)$ times implies that

$$
\Delta(n) \leq 2^{2^{c(K, D) \log ^{*}(n)},}
$$

as required.

\section{Proof of Theorem 1.2}

We shall now explain why the 2-convexification of the Tsirelson space $T^{(2)}$, as defined in the introduction, satisfies the $\mathrm{J}-\mathrm{L}$ lemma. First we give a definition. Given an increasing sequence $h(n)$ with $0 \leq h(n) \leq n$, we say that a Banach space $X$ is $h$-Hilbertian provided that for every finite-dimensional subspace $E$ of $X$, there are subspaces $F$ and $G$ of $E$ such that $E=F \oplus G, \operatorname{dim}(F)=O(h(\operatorname{dim} E))$, and $c_{2}(G)=O(1) .{ }^{1}$ If the Banach space $X$ is log-Hilbertian, then $X$ satisfies the $\mathrm{J}-\mathrm{L}$ lemma. Indeed, take $x_{1}, \ldots, x_{n} \in X$ and let $E$ be their span. Write $E=F \oplus G$ as above and decompose each of the $x_{i}$ accordingly, i.e., $x_{i}=y_{i} \oplus z_{i}$ where $y_{i} \in F$ and $z_{i} \in G$. Since $c_{2}(G)=O(1)$, by the $\mathrm{J}-\mathrm{L}$ lemma we can find a linear operator $L: G \rightarrow G^{\prime}$, where $G^{\prime} \subseteq G$ is a subspace of dimension $O(\log n)$, such that $\left\|z_{i}-z_{j}\right\|=\Theta\left(\left\|L\left(z_{i}\right)-L\left(z_{j}\right)\right\|\right)$ for all $i, j \in\{1, \ldots, n\}$. The linear operator $L^{\prime}: E \rightarrow F \oplus G^{\prime}$ given by $L^{\prime}(y \oplus z)=y \oplus L(z)$ has rank $O(\log n)$ and satisfies $\left\|x_{i}-x_{j}\right\|=\Theta\left(\left\|L^{\prime}\left(x_{i}\right)-L^{\prime}\left(x_{j}\right)\right\|\right)$, as required.

We now explain why $T^{(2)}$ satisfies the J-L lemma. In [22] Johnson defined the following modification of the Tsirelson space. As in the case of the Tsirelson space, the construction consists of an inductive definition of a sequence of norms on $c_{00}$. Once again we set $\|x\|_{0}=\|x\|_{c_{0}}$ and

$$
\begin{aligned}
\|x\|_{m+1}=\max \left\{\|x\|_{m}, \frac{1}{2} \sup \left\{\sum_{j=1}^{(n+1)^{n}}\left\|P_{A_{j}}(x)\right\| \|_{m}: n \in \mathbb{N},\right.\right. \\
\left.\left.A_{1}, \ldots, A_{(n+1)^{n}} \subseteq[n, \infty) \text { finite \& disjoint }\right\}\right\} .
\end{aligned}
$$

We then define $\|x\|_{\mathcal{T}}:=\lim _{m \rightarrow \infty}\|x\|_{m}$ and the modified space $\mathcal{T}^{(2)}$ as the completion of $c_{00}$ under the norm

$$
\left\|\sum_{j=1}^{\infty} x_{j} e_{j}\right\|_{\mathcal{T}^{(2)}}:=\left\|\sum_{j=1}^{\infty}\left|x_{j}\right|^{2} e_{j}\right\|_{\mathcal{T}}^{1 / 2} .
$$

In [23] Johnson proved that a certain subspace $Y$ of $\mathcal{T}^{(2)}$ (spanned by a subsequence of the unit vector basis) is log-Hilbertian. In [10] Casazza, Johnson, and Tzafriri showed that it is not necessary to pass to the subspace $Y$, and in fact $\mathcal{T}^{(2)}$

\footnotetext{
${ }^{1}$ Here the direct sum notation means as usual that for every $y \oplus z \in F \oplus G$, we have $\|y \oplus z\|_{X}=\Theta\left(\|y\|_{X}+\right.$ $\left.\|z\|_{X}\right)$, where the implied constants are independent of $E$.
} 
itself has the desired decomposition property. Finally, a deep result of Casazza and Odell [11] shows that $T^{(2)}$ is just $\mathcal{T}^{(2)}$ with an equivalent norm. This concludes the proof of the fact that $T^{(2)}$ satisfies the J-L lemma.

It remains to establish the lower bound (3). Note that the fact that $c_{2}\left(T^{(2)}\right)=\infty$ already follows from the original paper of Figiel and Johnson [16]—our goal here is to give a quantitative estimate. This will be a simple consequence of a paper of Bellenot [5]. Define inductively a sequence of functions $\left\{g_{k}: \mathbb{N} \rightarrow \mathbb{N}\right\}_{k=0}^{\infty}$ as follows: $g_{0}(n)=n+1$ and $g_{i+1}(n)=g_{i}^{(n)}(n)$, where $g_{i}^{(n)}$ denotes the $n$-fold iterate of $g_{i}$, i.e., $g_{i}^{(n+1)}(j)=g_{i}\left(g_{i}^{(n)}(j)\right)$. The inverse Ackermann function is the inverse of the function $n \mapsto g_{n}(n)$, i.e., its value on $n \in \mathbb{N}$ is the unique integer $k$ such that $g_{k}(k)<n \leq g_{k+1}(k+1)$. Note that in the literature there are several variants of the inverse Ackermann function, but it is possible to show that they are all the same up to bounded additive terms - see, for example, [2, Appendix B] for a discussion of such issues. In particular, we define $\alpha(n)$ to be the inverse of the function $h(n)=g_{n}(2)$, but its asymptotic behavior is the same as the inverse Ackermann function (since $g_{n}(2)>n$, and therefore $\left.g_{n}(n)<g_{n}\left(g_{n}(2)\right)=g_{n+1}(2)\right)$. Now, by [5, Proposition 5] for every $k \geq 1$, there exist scalars $\left\{x_{j}\right\}_{j=1}^{g_{k}(2)} \subseteq \mathbb{R}$ which are not all equal to 0 such that

$$
\left\|\sum_{j=1}^{g_{k}(2)} x_{j} e_{j}\right\|_{T} \leq \frac{k}{2^{k}} \sum_{j=3}^{g_{k}(2)}\left|x_{j}\right| .
$$

Hence, by the definition (5) we have for all $\varepsilon=\left(\varepsilon_{1}, \ldots, \varepsilon_{g_{k}(2)}\right) \in\{-1,1\}^{g_{k}(2)}$,

$$
\left\|\sum_{j=1}^{g_{k}(2)} \varepsilon_{j} x_{j} e_{j}\right\|_{T^{(2)}}^{2} \leq \frac{k^{2}}{2^{2 k}} \sum_{j=1}^{g_{k}(2)} x_{j}^{2} .
$$

Let $F \subseteq T^{(2)}$ denote the span of $\left\{e_{1}, \ldots, e_{g_{k}(2)}\right\}$. Averaging (30) over $\varepsilon$ and using the definition of the cotype 2 constant of $F$, we see that $C_{2}(F) \geq 2^{k} / k$, and therefore the Euclidean distortion of $F$ is at least $2^{k} / k$. Since the dimension of $F$ is $g_{2}(k)$, this concludes the proof of (3) and hence also the proof of Theorem 1.2.

\section{Remarks and Open Problems}

We end this note with some concluding comments and questions that arise naturally from our work.

1. The space $T^{(2)}$ was the first example of what Pisier [37, Chap. 12] calls weak Hilbert spaces. One of the many equivalents for a Banach space $X$ to be a weak Hilbert is that every finite-dimensional subspace $E$ of $X$ can be written as $E=F \oplus G$ with $\operatorname{dim} G \geq \delta \operatorname{dim} E$ for some universal constant $\delta>0$ and $c_{2}(G)=O(1)$. It is not known whether every weak Hilbert space is log-Hilbertian or even $h$-Hilbertian for some $h(n)=o(n)$. However, Nielsen and TomczakJaegermann [34], using the same kind of reasoning that works for $T^{(2)}$ (see [10]), proved that a weak Hilbert space with an unconditional basis is even $2^{O(\alpha(\cdot))}$ Hilbertian. 
2. A Banach space $X$ is called asymptotically Hilbertian provided that for each $n$ there is a finite-codimensional subspace $Y$ of $X$ such that $\Delta_{Y}(n)=O(1)\left(\Delta_{Y}(n)\right.$ is defined in (12)). Every weak Hilbert space is asymptotically Hilbertian [37, Chap. 14]. The results in [23] and the argument at the beginning of Sect. 3 show that every asymptotically Hilbertian space has a subspace which satisfies the J-L lemma.

3. Does there exist a function $f(n) \uparrow \infty$ such that if $X$ is a Banach space for which $\Delta(n)=O(f(n))$, where $\Delta(n)$ is as in (12), i.e., $c_{2}(E)=O(f(\operatorname{dim} E))$ for all finite-dimensional subspaces $E$ of $X$, then $X$ satisfies the J-L lemma? An affirmative answer would show that there are natural Banach spaces other than Hilbert spaces, even some Orlicz sequence spaces, which satisfy the J-L lemma.

4. A question which obviously arises from our results is to determine the true rate of "closeness" (in the sense of (2)) between spaces satisfying the J-L lemma and Hilbert space. Which of the bounds $\Delta(n)=2^{2^{O\left(\log ^{*}(n)\right)}}$ and $\Delta(n)=2^{\Omega(\alpha(n))}$ is closer to the truth?

5. Our argument also works when the dimension is only assumed to be reduced to a power of $\log n$, and we get nontrivial bounds even when this dimension is, say, $2^{(\log n)^{\beta}}$ for some $\beta<1$. However, except for spaces that are of type 2 or of cotype 2 , our proof does not yield any meaningful result when the dimension is lowered to $n^{\gamma}$ for some $\gamma \in(0,1)$. The problem is that in the recursive inequality (26) the term $\Delta(4 K \log (n+1))$ is squared. This happens since in Kwapien's theorem (8) the Euclidean distortion is bounded by the product of the type 2 and cotype 2 constants rather than by their maximum. While it is tempting to believe that the true bound in Kwapien's theorem should be $c_{2}(X)=O\left(\max \left\{T_{2}(X), C_{2}(X)\right\}\right)$, it was shown by Tomczak-Jaegermann [41, Proposition 2] that up to universal constants Kwapien's bound $c_{2}(X) \leq T_{2}(X) C_{2}(X)$ cannot be improved.

6. In [35] Pisier proved that if a Banach space $X$ satisfies $\Delta(n)=o(\log n)$, then $X$ is superreflexive; i.e., $X$ admits an equivalent norm which is uniformly convex. Hence any space satisfying the assumptions of Theorem 1.1 is superreflexive.

7. It is of interest to study dimension reduction into arbitrary low-dimensional normed spaces, since this can serve just as well for the purpose of data compression (see [32]). Assume that $X$ is a Banach space such that for every $n$ and $x_{1}, \ldots, x_{n} \in X$, there exists a $d(n)$-dimensional Banach space $Y$ and a linear mapping $L: X \rightarrow Y$ such that $\left\|x_{i}-x_{j}\right\| \leq\left\|L\left(x_{i}\right)-L\left(x_{j}\right)\right\| \leq D\left\|x_{i}-x_{j}\right\|$ for all $i, j \in\{1, \ldots, n\}$. Since by John's theorem [21] we have $c_{2}(Y) \leq \sqrt{d(n)}$, we can argue similarly to the proof in Sect. 2 (in this simpler case the proof is close to the argument in [30]), while using the result of Tomczak-Jaegermann (9), to deduce that $T_{2}(F), C_{2}(F) \leq 2 \pi \sqrt{d(n)}$. By Kwapien's theorem we deduce that $c_{2}(F) \leq 4 \pi^{2} d(n)$. If $d(n) \leq n^{\gamma}$ for some $\gamma \in(0,1)$ which is independent of $n$ and $F$, the fact that $T_{2}(F), C_{2}(F) \leq 2 \pi n^{\gamma / 2}$ for every $n$-dimensional subspace $F \subseteq X$ implies (see [42]) that $X$ has type $\frac{2}{1+\gamma}-\varepsilon$ and cotype $\frac{2}{1-\gamma}+\varepsilon$ for every $\varepsilon>0$. In particular, if $d(n)=n^{o(1)}$, then $X$ has type $2-\varepsilon$ and cotype $2+\varepsilon$ for every $\varepsilon>0$.

8. We do not know of any nontrivial linear dimension reduction result in $L_{p}$ for $p \in[1, \infty) \backslash\{2\}$. For example, is it possible to embed with $O(1)$ distortion via a linear mapping any $n$-point subset of $L_{1}$ into a subspace of $L_{1}$ of dimension, 
say, $n / 4$, or even into $\ell_{1}^{n / 4}$ ? Remarkably even such modest goals seem to be beyond the reach of current techniques. Clearly $n$-point subsets of $L_{1}$ are in their $n$ dimensional span, but we do not know if they embed with constant distortion into $\ell_{1}^{d}$ when $d=O(n)$. Schechtman proved in [38] that we can take $d=O(n \log n)$. We refer to $[8,26,38,39]$ for more information on the harder problem of embedding $n$-dimensional subspaces of $L_{1}$ into low-dimensional $\ell_{1}^{d}$. We also refer to these references for similar results in $L_{p}$ spaces.

\section{References}

1. Ailon, N., Chazelle, B.: Approximate nearest neighbors and the fast Johnson-Lindenstrauss transform. In: STOC 2006: ACM Symposium on Theory of Computing, pp. 557-563 (2006)

2. Alon, N., Kaplan, H., Nivasch, G., Sharir, M., Smorodinsky, S.: Weak $\epsilon$-nets and interval chains. In: Proceedings of the Nineteenth Annual ACM-SIAM Symposium on Discrete Algorithms, SODA 2008, San Francisco, California, USA, January 20-22, 2008, pp. 1194-1203. SIAM, Philadelphia (2008)

3. Arias-de Reyna, J., Rodríguez-Piazza, L.: Finite metric spaces needing high dimension for Lipschitz embeddings in Banach spaces. Isr. J. Math. 79(1), 103-111 (1992)

4. Ball, K.: An elementary introduction to modern convex geometry. In: Flavors of Geometry. Math. Sci. Res. Inst. Publ., vol. 31, pp. 1-58. Cambridge Univ. Press, Cambridge (1997)

5. Bellenot, S.F.: The Banach space $T$ and the fast growing hierarchy from logic. Isr. J. Math. 47(4), 305-313 (1984)

6. Benyamini, Y., Lindenstrauss, J.: In: Geometric Nonlinear Functional Analysis. Vol. 1. American Mathematical Society Colloquium Publications, vol. 48. American Mathematical Society, Providence (2000)

7. Bourgain, J.: On Lipschitz embedding of finite metric spaces in Hilbert space. Isr. J. Math. 52(1-2), 46-52 (1985)

8. Bourgain, J., Lindenstrauss, J., Milman, V.: Approximation of zonoids by zonotopes. Acta Math. 162(1-2), 73-141 (1989)

9. Brinkman, B., Charikar, M.: On the impossibility of dimension reduction in $l_{1}$. J. ACM 52(5), 766788 (2005) (electronic)

10. Casazza, P.G., Johnson, W.B., Tzafriri, L.: On Tsirelson's space. Isr. J. Math. 47(2-3), 81-98 (1984)

11. Casazza, P.G., Odell, E.: Tsirelson's space and minimal subspaces. In: Texas Functional Analysis Seminar 1982-1983 (Austin, TX). Longhorn Notes, pp. 61-72. Univ. Texas Press, Austin (1983)

12. Casazza, P.G., Shura, T.J.: Tsirel'son's Space. Lecture Notes in Mathematics, vol. 1363. Springer, Berlin (1989). With an appendix by J. Baker, O. Slotterbeck, and R. Aron

13. Charikar, M., Sahai, A.: Dimension reduction in the $\ell_{1}$ norm. In: 43rd Annual IEEE Conference on Foundations of Computer Science, pp. 251-260. IEEE Computer Society, Los Alamitos (2002)

14. Cirel'son, B.S.: It is impossible to imbed $1_{p}$ of $c_{0}$ into an arbitrary Banach space. Funkt. Anal. Prilozh. 8(2), 57-60 (1974)

15. Danzer, L., Grünbaum, B., Klee, V.: Helly's theorem and its relatives. In: Proc. Sympos. Pure Math., vol. VII, pp. 101-180. Am. Math. Soc., Providence (1963)

16. Figiel, T., Johnson, W.B.: A uniformly convex Banach space which contains no $l_{p}$. Compos. Math. 29, 179-190 (1974)

17. Figiel, T., Lindenstrauss, J., Milman, V.D.: The dimension of almost spherical sections of convex bodies. Acta Math. 139(1-2), 53-94 (1977)

18. Indyk, P.: Algorithmic applications of low-distortion geometric embeddings. In: 42nd IEEE Symposium on Foundations of Computer Science, Las Vegas, NV, 2001, pp. 10-33. IEEE Computer Soc., Los Alamitos (2001)

19. Indyk, P.: Stable distributions, pseudorandom generators, embeddings, and data stream computation. J. ACM 53(3), 307-323 (2006) (electronic)

20. Indyk, P., Motwani, R.: Approximate nearest neighbors: towards removing the curse of dimensionality. In: STOC '98 (Dallas, TX), pp. 604-613. ACM, New York (1999)

21. John, F.: Extremum problems with inequalities as subsidiary conditions. In: Studies and Essays Presented to R. Courant on his 60th Birthday, January 8, 1948, pp. 187-204. Interscience, New York (1948) 
22. Johnson, W.B.: A reflexive Banach space which is not sufficiently Euclidean. Stud. Math. 55(2), 201205 (1976)

23. Johnson, W.B.: Banach spaces all of whose subspaces have the approximation property. In: Special Topics of Applied Mathematics, Proc. Sem., Ges. Math. Datenverarb., Bonn, 1979, pp. 15-26. NorthHolland, Amsterdam (1980)

24. Johnson, W.B., Lindenstrauss, J.: Extensions of Lipschitz mappings into a Hilbert space. In: Conference in Modern Analysis and Probability (New Haven, Conn., 1982). Contemp. Math., vol. 26, pp. 189-206. Am. Math. Soc., Providence (1984)

25. Johnson, W.B., Lindenstrauss, J., Schechtman, G.: On Lipschitz embedding of finite metric spaces in low-dimensional normed spaces. In: Geometrical Aspects of Functional Analysis (1985/86). Lecture Notes in Math., vol. 1267, pp. 177-184. Springer, Berlin (1987)

26. Johnson, W.B., Schechtman, G.: Finite dimensional subspaces of $L_{p}$. In: Handbook of the Geometry of Banach Spaces, vol. I, pp. 837-870. North-Holland, Amsterdam (2001)

27. Kleinberg, J.M.: Two algorithms for nearest-neighbor search in high dimensions. In: STOC '97 (El Paso, TX), pp. 599-608. ACM, New York (1999) (electronic)

28. Kushilevitz, E., Ostrovsky, R., Rabani, Y.: Efficient search for approximate nearest neighbor in high dimensional spaces. SIAM J. Comput. 30(2), 457-474 (2000) (electronic)

29. Kwapień, S.: Isomorphic characterizations of inner product spaces by orthogonal series with vector valued coefficients. Stud. Math. 44, 583-595 (1972). Collection of articles honoring the completion by Antoni Zygmund of 50 years of scientific activity, VI

30. Lee, J.R., Mendel, M., Naor, A.: Metric structures in $L_{1}$ : dimension, snowflakes, and average distortion. Eur. J. Combin. 26(8), 1180-1190 (2005)

31. Lee, J.R., Naor, A.: Embedding the diamond graph in $L_{p}$ and dimension reduction in $L_{1}$. Geom. Funct. Anal. 14(4), 745-747 (2004)

32. Matoušek, J.: Bi-Lipschitz embeddings into low-dimensional Euclidean spaces. Comment. Math. Univ. Carol. 31(3), 589-600 (1990)

33. Matoušek, J.: On the distortion required for embedding finite metric spaces into normed spaces. Isr. J. Math. 93, 333-344 (1996)

34. Nielsen, N.J., Tomczak-Jaegermann, N.: Banach lattices with property (H) and weak Hilbert spaces. Ill. J. Math. 36(3), 345-371 (1992)

35. Pisier, G.: Martingales with values in uniformly convex spaces. Isr. J. Math. 20(3-4), 326-350 (1975)

36. Pisier, G.: Factorization of Linear Operators and Geometry of Banach Spaces. CBMS Regional Conference Series in Mathematics, vol. 60. Conference Board of the Mathematical Sciences, Washington (1986)

37. Pisier, G.: The Volume of Convex Bodies and Banach Space Geometry. Cambridge Tracts in Mathematics, vol. 94. Cambridge University Press, Cambridge (1989)

38. Schechtman, G.: More on embedding subspaces of $L_{p}$ in $l_{r}^{n}$. Compos. Math. 61(2), 159-169 (1987)

39. Talagrand, M.: Embedding subspaces of $L_{1}$ into $l_{1}^{N}$. Proc. Am. Math. Soc. 108(2), 363-369 (1990)

40. Tomczak-Jaegermann, N.: Computing 2-summing norm with few vectors. Ark. Mat. 17(2), 273-277 (1979)

41. Tomczak-Jaegermann, N.: The Banach-Mazur distance between symmetric spaces. Isr. J. Math. 46(12), 40-66 (1983)

42. Tomczak-Jaegermann, N.: Banach-Mazur Distances and Finite-Dimensional Operator Ideals. Pitman Monographs and Surveys in Pure and Applied Mathematics, vol. 38. Longman, Harlow (1989)

43. Vempala, S.S.: The Random Projection Method. DIMACS Series in Discrete Mathematics and Theoretical Computer Science, vol. 65. American Mathematical Society, Providence (2004). With a foreword by Christos H. Papadimitriou 